Philip Thomas

\title{
Vaccine-mediated exit strategies from England's Covid-19 lockdown
}

ABSTRACT. An assessment is made of vaccine-mediated exit strategies from England's Covid-19 lockdown. Two linked methods are considered. The first, termed the gradualist approach, assumes that the R-rate is controlled to a value close to 1.0 and that the social distancing index (SDI), which measures the nation's level of interpersonal contact, is allowed to increase continuously to the point where it can grow no further and society and the economy are fully open. The second method, termed the two-step strategy, adopts the gradualist approach initially, but then, after a certain time, the SDI is stepped to its maximum value, which implies the immediate removal of all restrictions. It is found that, while vaccination-generated immunity makes a very valuable contribution to overall immunity, the other components-prior T-cell immunity and immunity generated by infection - are just as important. Infection-generated immunity needs to be the largest component if all restrictions are to be removed, under both strategies. Leaving lockdown to the point where all restrictions can be fully eased requires a narrow path to be followed during the spring and early summer of 2021, keeping the R-rate in a central band around 1.0. Close control of the R-rate is needed and this will require it to be measured accurately, continuously and rapidly. The gradualist approach might allow all restrictions to be lifted by the end of the summer 2021, while the two-step strategy might offer the prospect of full derestriction by the end of May 2021.

Keywords: coronavirus, Covid-19, lockdown, lockdown exit strategies, predictor-corrector coronavirus filter, PCCF

Nanotechnology Perceptions 17 (2021) 30-73

doi: 10.4024/N02TH21A.ntp.17.01 